\title{
MODELOS AMPLIADOS DIGITALES PARA PLANIFICACIÓN Y GESTIÓN DE INTERVENCIONES SOBRE EDIFICIOS $Y$ ENTORNOS URBANOS COMPLEJOS.
}

\author{
J.J.Fernández, J.Finat, L.Fuentes, J.Martínez, J.D.Pérez-Moneo, J.I.San \\ José, J.Tordable
}

Laboratorio de Fotogrametría Arquitectónica, ETS de Arquitectura, 47014 Valladolid

Lab MoBiVAP 2.2 Edificio I+D, Campus M. Delibes, 47011 Valladolid

Grupo DAVAP, Universidad de Valladolid, Valladolid, España.

juanjo@ega.uva.es, jfinat@agt.uva.es, Ifuentes@fa1.uva.es, jmr@ega.uva.es, juandi@ega.uva.es, jesusanjose@ono.com, jtordable@dyriad.com

Remisión Artículo: 15-5-2007

Palabras Clave: Fotogrametría terrestre, Visión Computacional y láser 3d, modelos de Realidad Virtual

Resumen: Los recientes avances en la captura y procesamiento de información 3d permiten abordar la elaboración y gestión de documentación a diferentes niveles de resolución para aplicarlo en tareas de documentación y visualización. El sistema desarrollado por el grupo DAVAP de la Universidad de Valladolid, combina metodologías basadas en fotogrametría terrestre, Visión Computacional y láser $3 \mathrm{~d}$. La combinación de todos ellos proporciona un soporte para la elaboración de modelos de Realidad Virtual y Aumentada sobre entornos generados según las metodologías precedentes. En este trabajo se exponen los modelos virtuales desarrollados para interior de edificios (Teatro Zorrilla de Valladolid) ${ }^{1}$, entornos arqueológicos aumentados (Teatro Romano de Clunia $^{2}$ ) y escenarios urbanos complejos (Muralla de León y el Área de Rehabilitación Integral -ARI- Tierras del renacimiento ${ }^{3}$ ).

La referencia volumétrica de los modelos generados procede del pegado de nubes densas de puntos $3 d$ capturadas por el dispositivo láser Ilris $3 d$ de Optech. La nube densa obtenida tras el pegado de diferentes tomas y optimizada proporciona un entorno global preciso como modelo de referencia para la incorporación de información procedente de vistas y evaluación de propiedades métricas. El soporte digital obtenido permite la generación y visualización de primitivas geométricas exportables a CAD.

\footnotetext{
${ }^{1}$ Elaborado mediante un Convenio entre la Universidad de Valladolid y la Diputación de Valladolid.

${ }^{2}$ Trabajo circunscrito dentro de una marco de Colaboración entre los Directores del Plan Director de la Colonia de Clunia Sulplicia y el grupo LFA-DAVAP de la Universidad de Valladolid.

${ }^{3}$ El trabajo sobre las Murallas de León ha sido financiado por el ILRUV Instituto Leones de la Restauración Urbana y Vivienda y por los arquitectos Ramón Cañas y Carlos Sexmilo. El proyecto ARI Tierras de Renacimiento ha sido financiado por la Junta de Castilla y León y el consorcio de Ayuntamientos de la Nava. En estos trabajos han intervenido además de los autores: Luís Antonio García García; Ana Isabel Gómez Olivar; Tomás González Martínez; David Marcos González; Francisco M. Morillo y Maribel Sánchez.
} 


\section{INTRODUCCIÓN}

La generación de modelos 3d puede realizarse de forma sintética ó analítica. La generación sintética de modelos utiliza herramientas de diseño asistido y primitivas geométricas como piezas básicas a partir de las cuales se generan objetos ó escenarios más complejos; la comparación entre modelos generados de forma sintética se lleva a cabo de forma interactiva mediante las herramientas de ajuste y modelado 3d. La generación analítica de modelos usa herramientas de procesamiento sobre datos digitales (imágenes ó escaneos). El procesamiento de la información se lleva a cabo mediante herramientas de filtrado, selección y agrupamiento en torno a datos significativos (contornos/bordes y superficies típicamente); la comparación entre modelos generados de forma analítica se lleva a cabo mediante ajuste de haces (fotogrametría) ó bien mediante la introducción de restricciones geométricas (restricción epipolar) ó radiométricas (consistencia local en vóxels).

Los modelos generados de forma sintética son más ligeros y su manipulación presenta un menor coste computacional; resultan más apropiados en tareas de visualización. Por el contrario, los modelos generados de forma analítica presentan una mayor precisión y son más apropiados en tareas de documentación. El incremento de la capacidad de procesamiento de ordenadores de sobremesa y las necesidades de trabajar con diferentes niveles de detalle ha motivado el desarrollo de modelos multirresolución.

Ello requiere desarrollar herramientas software que permitan compatibilizar las aproximaciones sintética y analítica. Existe un gran número de conexiones entre ambas aproximaciones que facilitan el transvase de resultados. Para entender la complejidad de este transvase es preciso entender que la diferencia fundamental entre las aproximaciones sintética y analítica, es el carácter continuo y discreto de los inputs con los que se trabaja (primitivas geométricas/topológicas ó nubes de puntos 2d/3d contenidos en imágenes/escaneos4).

El enfoque sintético se puede discretizar introduciendo herramientas de muestreo sobre los objetos generados y desarrollando métodos de compresión de las estructuras geométricas ó topológicas generadas (mallas, típicamente). Para dotar de un soporte continuo a los objetos generados según la estrategia analítica, es necesario introducir estructuras superpuestas lineales a trozos (piecewise linear ó PL en lo sucesivo) ó lisas que se ajusten de forma óptima a los datos discretos disponibles. La diversidad de objetos reales da lugar a que no exista un único funcional que permita ajustar de forma simultánea los datos discretos a objetos continuos; por ello, se utilizan funcionales híbridos y se modelan/implementan procedimientos de optimización multiobjetivo con una fuerte componente interactiva para dotar de pesos variables a las características geométricas, radio métricas y estadísticas de los datos discretos.

Para resolver este problema, se ha desarrollado la plataforma software UVACAD (Utilidad de Visualización Avanzada Con Automatización de Dibujo) que facilita las operaciones proyectivas básicas (secciones y proyecciones) relativas a edificios y entornos urbanos a partir de nubes de puntos. Asimismo, se han implementado herramientas software para la reproyección de fotos de alta resolución sobre los modelos $3 d$ densos. De este modo, es posible incorporar detalles relativos a modelos métricos a diferente resolución. La utilización de UVACAD permite realizar consultas sobre edificios ó entornos urbanos acotados, proporcionando un primer paso para Sistemas de Información Urbanos a alta resolución. Asimismo, la documentación 3d generada proporciona un soporte para la superposición de modelos virtuales sobre los escenarios $3 d$ generados que resulta especialmente útil para planificación y recuperación de entornos urbanos.

Este trabajo ilustra la metodología seguida por el grupo DAVAP (Documentación, Análisis y Visualización Avanzada del Patrimonio) de acuerdo con el esquema siguiente: En la sección 2 se aborda el modelado basado en láser $3 d$. 
En la sección 3 se esbozan algunas líneas relacionadas con los sistemas híbridos de información 3d basados en cartografía digital, fotografía y láser 3d.

La sección 4 aborda el problema de la generación semi-automática de entornos virtuales basada en una reducción drástica de la información 3d proporcionada por el láser.

La sección 5 plantea algunas de las líneas que se están iniciando en relación con la accesibilidad y gestión de la información 3d basada en láser 3d para un SIG urbano a pequeña escala.

Por último, en el apartado 6 Trabajo en curso, se esbozan algunas de las líneas principales en las que se está avanzando para una gestión integral de información.

\section{MODELADO A GRAN ESCALA BASADO EN LÁSER.}

El modelado $3 d$ de edificios y entornos urbanos puede ir orientado hacia tareas de documentación ó de visualización, según el propósito y la escala a la que opera5.

- El modelado 3d orientado a la documentación tiene un carácter exhaustivo y requiere una elevada precisión que se adapta bien a edificios aislados. Los archivos de puntos $3 d$ procedentes de una estación total y, en mayor mediad, los procedentes de un láser terrestre proporcionan información métrica densa que satisface los requerimientos de precisión en trabajos de documentación. Se dice que es una aproximación basada en el rango.

- El modelado orientado a la visualización tiene un carácter genérico y requiere facilidades de navegación y consulta; este modelado se adapta bien a entornos urbanos [3]. La información proporcionada por cartografía digital ó por cientos de fotos facilita la visualización mediante técnicas de Reconstrucción 3d (utilización de la geometría epipolar) ó de Informática Gráfica (incluyendo herramientas de realidad virtual sobre la cartografía digital como la extrusión, p.e.). Se dice que es una aproximación basada en la imagen (analítica ó sintética).

Ambos tipos de modelado 3D pueden ser sintéticos ó analíticos, es decir, pueden ser generados a partir de primitivas geométricas ó bien reconstruidos a partir de datos contenidos en imagen/escaneo láser. El modelado sintético se beneficia de la existencia de representaciones paramétricas y de un soporte continuo que simplifica la radiometría real de los objetos; por ello, la interacción se sitúa en los aspectos de renderización y está más orientado hacia la visualización. El modelado analítico se beneficia de la captura automática de la información; por ello, la interacción se sitúa en los aspectos del tratamiento de la información (filtrado, agrupamiento, extracción, restauración, análisis). A continuación se muestran ejemplos correspondientes a la aproximación sintética para visualizar el interior de edificios (Teatro Zorrilla de Valladolid) y a la aproximación analítica en entornos arqueológicos aumentados (Teatro Romano de Clunia ) y escenarios urbanos complejos (Muralla de León).

Figura 1: Teatro Zorrilla de Valladolid

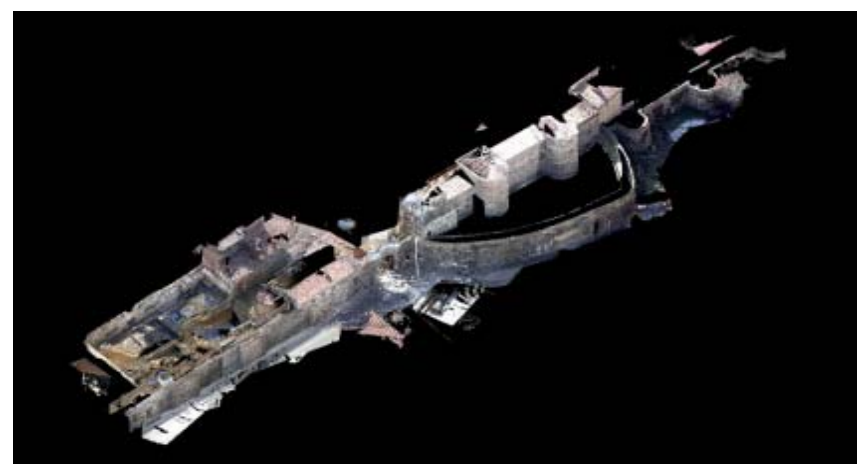


Figura 2: Teatro Romano de Clunia

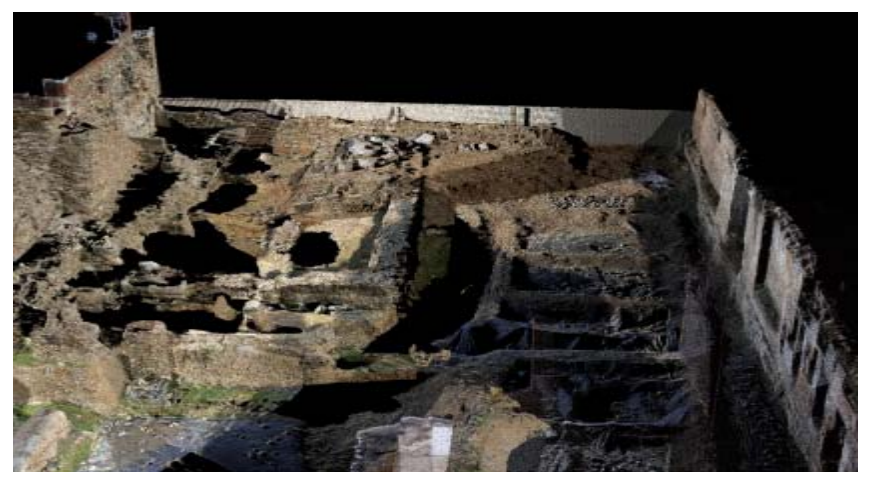

Existe una zona de León en el que se pueden apreciar las cuatro murallas existentes. El proyecto denominado 4 murallas, pretende una documentación real para la documentación del proyecto de intervención, una reconstrucciónideal para la visualización y divulgación y una reconstrucción virtual para la memoria. En definitiva mostramos lo que hay, lo que hubo y lo que queremos que haya. El pasado el presente y futuro unidos en un universo digital.

En principio, los propósitos, el nivel de detalle y la metodología del modelado orientado hacia la documentación y la visualización parecen incompatibles. Sin embargo, en los últimos años está teniendo lugar un acercamiento entre la documentación y la visualización utilizando modelos muestreados a baja resolución basados en láser 3d para visualización y modelos de Reconstrucción 3d densa basados en múltiples vistas para documentación. La multiresolución proporciona el marco para el transvase de dicha información.

Los modelos basados en láser pueden ser discretos ó continuos. Los modelos discretos a baja resolución se basan en la reducción de la nube de puntos mediante técnicas de muestreo regular, aleatorio ó, más recientemente dependiendo de funciones de importancia. Los modelos continuos utilizan una estructura superpuesta continua basada en la nube de puntos de tipo lineal a trozos ó PL.estructura (una malla regular ó irregular) ó bien un suavizado de dicha PLestructura. La existencia de un soporte continuo para estructuras lineales a trozos ó bien lisas, facilita la simplificación del modelo mediante la identificación de primitivas geométricas asociadas a la PL. estructura.

Asimismo, la implementación de algoritmos de propagación sobre el soporte proporcionado por la estructura continua (PL ó lisa) permite restaurar los archivos digitales originales (de imagen ó de rango) y evitar artefactos asociados a la captura ó al procesamiento de vistas ó de escaneo, proporcionando una visualización más compacta.

\section{HERRAMIENTAS DE GESTIÓN}

El paisaje urbano es el resultado de una interacción entre aspectos históricos, socioeconómicos, políticos y culturales. El modelado a gran escala del paisaje urbano proporciona un soporte físico para analizar y entender las relaciones físicas, funcionales y socio-económicas entre los diferentes aspectos. Por ello, las herramientas de gestión deben contribuir a la comprensión de las complejas interacciones entre factores y comportamientos, proponiendo soluciones para modificar los efectos perversos en áreas desarrolladas y contribuyendo a prevenir la falta de funcionalidad de actuaciones parciales mediante la simulación.

La consecución de estos objetivos requiere el desarrollo de modelos integrados de gestión que faciliten el análisis, el planeamiento, la simulación y el seguimiento de las políticas urbanas orientadas a mejorar la calidad de vida de los ciudadanos, con una especial atención al Patrimonio Cultural. La gestión de espacios urbanos debe ir orientada hacia la protección, conservación y puesta en valor de activos patrimoniales, mediante el desarrollo de una política 
integral de conservación. Una gestión integral avanzada basada en Tecnologías de la Información no sólo es más eficiente y facilita una mayor satisfacción a los ciudadanos, sino que también opera como un factor de dinamización cultural y económica, generando productos y servicios con un mayor valor añadido. La herramienta fundamental para la gestión de espacios urbanos es un SIP urbano.

Un Sistema de Información Patrimonial (SIP, en lo sucesivo), es un sistema de hardware, software y procedimientos diseñados para soportar la captura, la gestión, el análisis, el modelado y la presentación de datos espacialmente geo-referenciados de cara a resolver problemas de diseño, planificación, gestión y visualización. Un SIP se puede ver como:

- Una Aplicación Automatizada para producir mapas ó representaciones, es decir, una aplicación orientada a la generación automática de una asignación entre inputs y outputs.

- Un Inventario que posibilite la capacidad de acceso directo a bases de datos existentes con protocolos para el procesamiento de información y búsquedas por contenido.

- Un Soporte para el Análisis Espacial y Toma de Decisiones facilitando nuevos usos a partir de las bases de datos proporcionando a los usuarios herramientas de búsqueda y análisis.

- Un módulo para el Procesamiento y Análisis de la Información, incluyendo la posibilidad de generación interactiva de informes adaptada a los requerimientos del usuario de la aplicación.

- Un módulo de Visualización que permite comprender, analizar y explicar la distribución de objetos en el entorno territorial, que será cada vez más importante conforme el volumen de datos espaciales digitales vaya siendo mayor.

El grupo DAVAP de la Universidad de Valladolid juntamente con Labein y la empresa Global PC de Salamanca, han desarrollado un SIP urbano sobre 4 pueblos de la provincia de Palencia (España). La elección del programa comercial ArcGIS responde a la necesidad de entregar un producto a la Junta de Castilla y León y a los Ayuntamientos de la provincia de Palencia basado en una herramienta de uso común por parte de dichas instituciones.

La fase del análisis previo concierne al diseño de: a) la estructura de datos espaciales y b) los interfaces apropiados para resolver las cuestiones de visualización y definición de los procesos de búsqueda de acuerdo con los requerimientos del cliente.

El desarrollo de la aplicación informática basada en ArcGIS se inicia con una normalización previa de la base de datos existente procedente del catastro para garantizar el correcto mantenimiento de la misma y el correcto acceso a los datos, procediendo a continuación a la reconversión de los datos originales a la nueva base de

datos. A continuación se ha desarrollado una aplicación que permite vincular la base cartográfica procedente de un Sistema de Información Geográfico con una base de datos externa que facilite los procesos de búsqueda basados en criterios almacenados en clave alfanumérica. La implementación de esta aplicación ha permitido desarrollar una cartografía temática y vincular (mediante tablas y relaciones) las características catastrales ó de identificación fiscal con información planimétrica, fotográfica y procedente del escaneo láser $3 d$ de dichos pueblos. En una fase más avanzada está prevista la implementación de métodos de reconocimiento, aprendizaje y búsqueda por contenido en $2 \mathrm{~d}$ y $3 \mathrm{~d}$. 


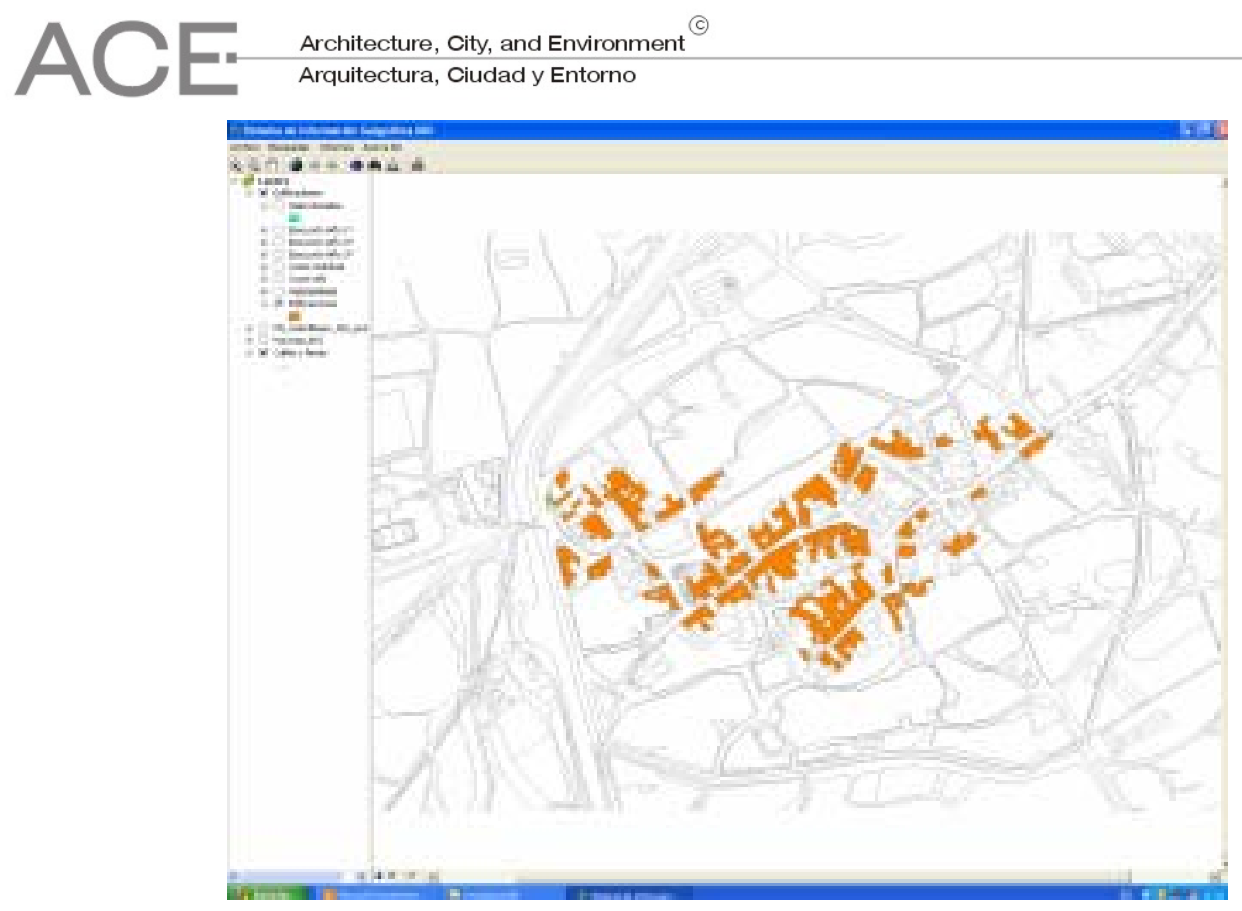

El menú de la izquierda Permite Seleccionar diferentes categorías de forma interactiva cliqueando con el puntero sobre el recuadro que está en blanco) que se visualizan con el mismo color. En el caso de "Ejecución" y "Coste Solicitud" como todavía no hay datos asociados no se resaltará ningún edificio.

La aplicación informática desarrollada permite abrir proyecto (uno para cada pueblo), visualizar los datos y generar informes (configurar, guardas, imprimir, salir) según datos catastrales y según diferentes tipos de intervenciones a realizar en cada uno de los edificios considerados, incluyendo el coste económico de las intervenciones realizadas.

La información se ha organizado en cuatro bases de datos independientes (una por cada pueblo), incluyendo procesos de búsqueda asociadas a Presupuestos planeados según valoración de la intervención, Reparaciones (necesarias ó efectuadas), Propietarios, Tipo de Ocupación (habitado, deshabitado, temporal). Asimismo, se han desarrollado los vínculos que permiten visualizar la información contenida en la cartografía temática y en la información fotográfica $2 d$ ó de escaneo láser $3 d$. La gestión es similar a la de la mayor parte de los programas basados en interfaces gráficos.
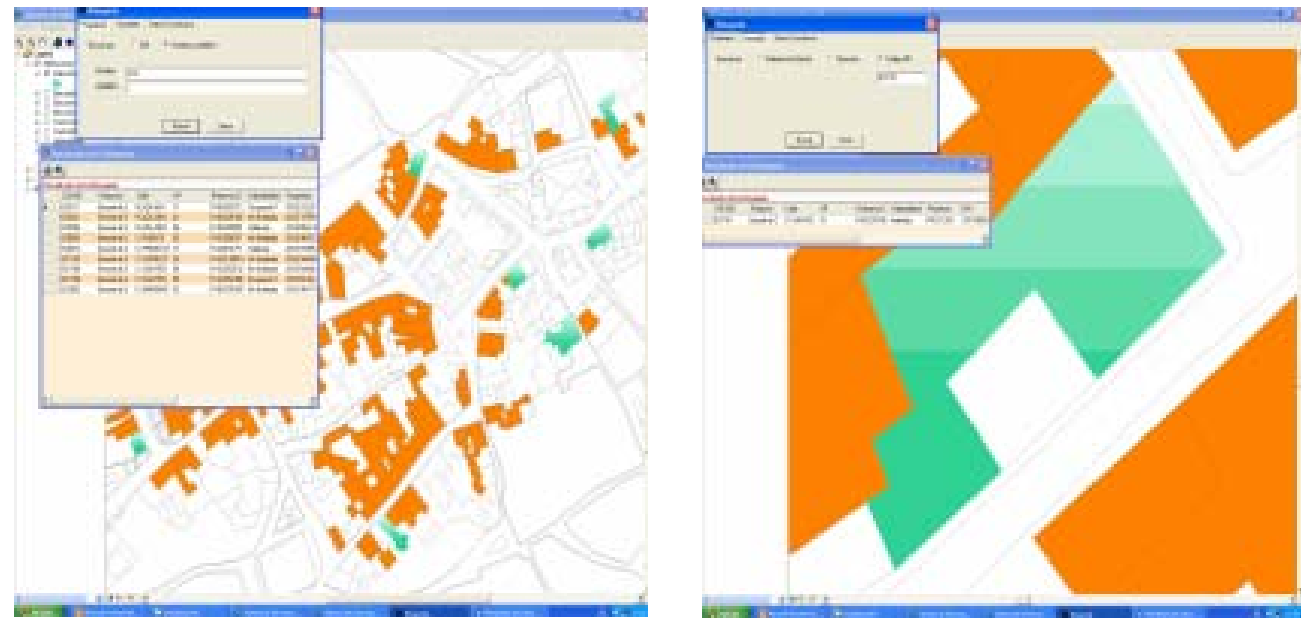
La aplicación desarrollada permite hacer búsquedas por datos personales, del inmueble ó económicos, generar un listado con los datos encontrados e imprimirlo. Asimismo, se dispone de herramientas de navegación sobre la cartografía digital coloreada, incluyendo ampliaciones.

Asimismo, la selección de la opción "Otros datos" permite acceder a los archivos que incluyen la información 2d/3d correspondiente a vistas/escaneos, con enlaces a Adobe Acrobat y UVACAD para su visualización. Para cada archivo seleccionado, el Programa UVACAD permite realizar una navegación $3 d$ sobre el modelo, una selección de una ventana a diferentes resoluciones y extracción de información métrica.
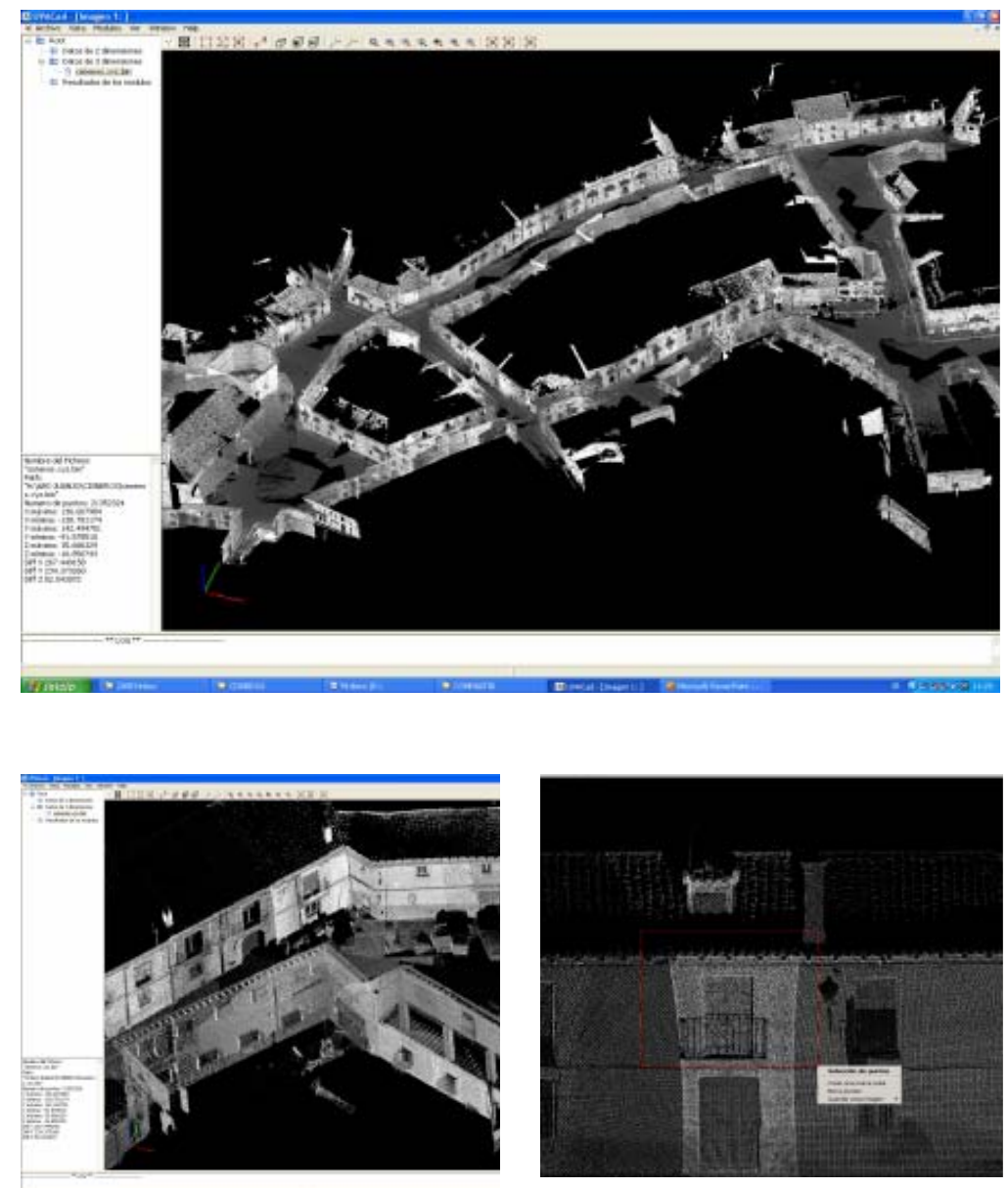

En la parte superior se proporciona la visualización del modelo $3 d$ asociado a una zona del pueblo y procedente de una segmentación volumétrica del modelo global $3 d$.

En la parte inferior derecha se visualiza un edificio en concreto, en una ventana más reducida con una orientación y resolución menor seleccionadas por el usuario sobre la que se realiza una consulta interactiva relativa a propiedades métrica. 


\section{GENERACIÓN SEMI-AUTOMÁTICA DE ENTORNOS VIRTUALES.}

Los entornos virtuales proporcionan una presentación e interacción multimodal basada en la integración de tecnologías que facilita al usuario la navegación, exploración y manipulación de objetos en un entorno sintético generado mediante herramientas computacionales. El carácter sintético del entorno debe proceder de una simplificación de la realidad modelada según métodos basados en cartografía, imagen ó rango.

Los métodos basados en cartografía digital son los más frecuentes. La extrusión de edificios mediante prismas rectangulares a partir de un plano digital utilizando la información catastral es una herramienta común en gran número de aplicaciones informáticas comerciales. La gestión de la navegación visual en torno a volúmenes extruidos correspondientes a edificios se apoya en implementar la restricción de visibilidad 3d para posiciones cambiantes del cliente. La visibilidad basada en intersecciones de conos proyectantes presenta un coste computacional elevado. Por ello, es preferible utilizar grafos dinámicos que faciliten una representación simbólica y facilidades para la gestión de la memoria asociada a amplios entornos.

La digitalización de cartografía histórica proporciona un soporte para el diseño de escenarios 2.5D en los que se han insertado a) alzados sobre el plano con propósitos de visualización de zonas que han sufrido transformaciones urbanas irreversibles (Plaza del Rosarillo de Valladolid, p.e.) ó bien b) modelos detallados basados en el Láser Ilris 3d de Optech con propósitos de documentación (entorno de la Plaza Mayor de Soria, p.e.).

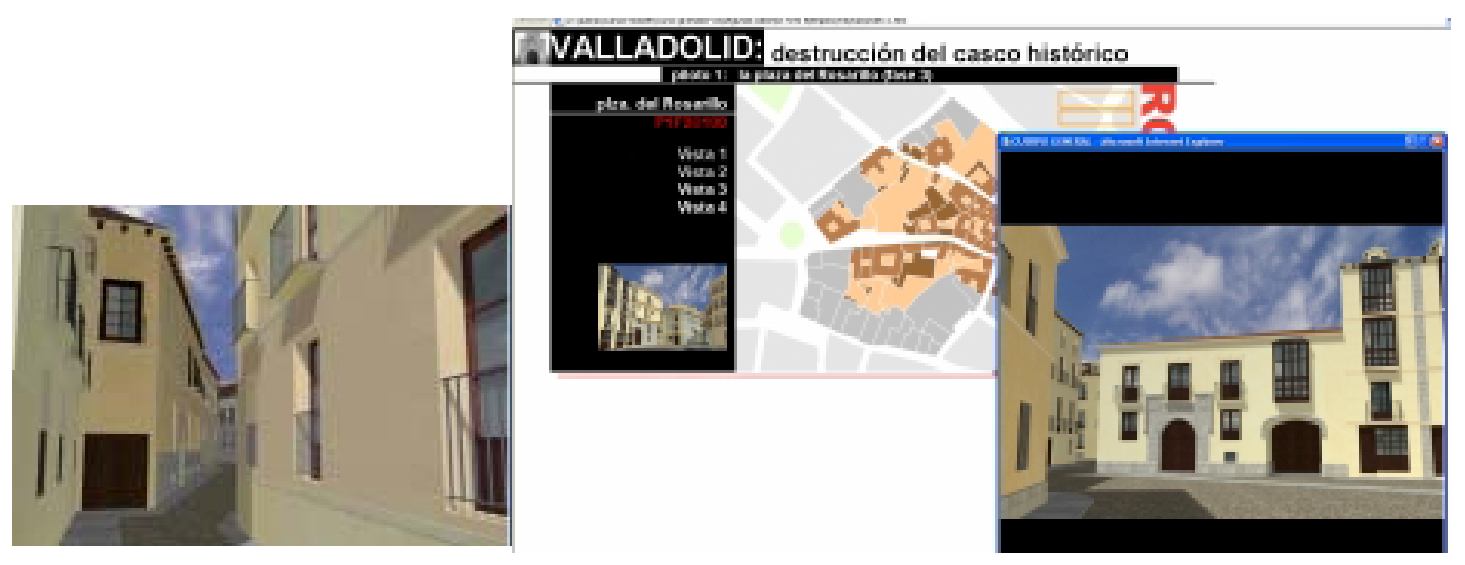

Los métodos basados en Visión Computacional permiten dotar de una apariencia más realista a los entornos virtuales, mediante la superposición de objetos $3 \mathrm{~d}$ y texturas a los modelos procedentes de la extrusión. Los métodos basados en Visión Computacional están siendo aplicados en tareas de visualización realista de entornos urbanos utilizando métodos basados en Reconstrucción 3d a partir de una gran cantidad de vistas (ver [4], [5], [6] y referencias allí citadas). La incorporación de un módulo de reproyección a la plataforma software UVACAD combina las aproximaciones basadas en Visión Computacional y en láser $3 \mathrm{~d}$, reproyectando fotos de alta resolución sobre nubes desnivel de detalle que resulta crucial para documentación de edificios a escala 1:20. 


\section{INTEGRACIÓN DE ENTORNOS VIRTUALES CON SIP}

La integración de entornos virtuales con SIP requiere el diseño e implementación de interfaces en un módulo de visualización avanzada que sea compatible con las funciones básicas de entornos virtuales (navegación, exploración y manipulación de objetos) y con las herramientas de los SIP (consulta, procesamiento y análisis de la información asociadas a las bases de datos).

El diseño de interfaces debe realizarse de acuerdo con sistemas de diálogo entre el Cliente y el Sistema de Consulta:

- El Cliente proporciona la base del proyecto a realizar y debe proporcionar los objetivos y la utilización que se dará a los datos en el futuro.

- El Sistema de Consulta debe mostrar claramente al cliente las diferentes posibilidades de la aplicación y los diferentes usos que se pueden dar a la información procesada.

Hay diferentes tipos de clientes privados y públicos, que incluyen profesionales (arquitectos, ingenieros civiles, expertos en Patrimonio, historiadores, expertos en Geografía Urbana, expertos en planificación, economistas), entidades locales (administrativas, culturales, agentes del catastro), agentes económicos y sociales (empresarios, agentes de relaciones públicas ó de agencias inmobiliarias), etc.

La aplicación a desarrollar debe ser lo bastante flexible como para adaptarse de forma satisfactoria a los requerimientos de todo tipo de clientes.

La e-accesibilidad a los servicios proporcionados por la administración y algunas empresas es un prerrequisito para diferentes formas de acceso remoto (según protocolos preestablecidos) a información estructurada a través de Internet. Uno de los ejemplos más completos se presenta Un ejemplo particularmente interesante concierne a la documentación $3 d$ de escenarios urbanos complejos, actualmente en proceso de redefinición, como el que corresponde a las Murallas Romanas y Cercas Medievales de León cuya visualización se muestra más abajo. La documentación llevada a cabo sobre el entorno actual proporciona un soporte para ensayar diferentes soluciones para la recuperación de un espacio urbano singular degradado y su reconversión a centro cultural.

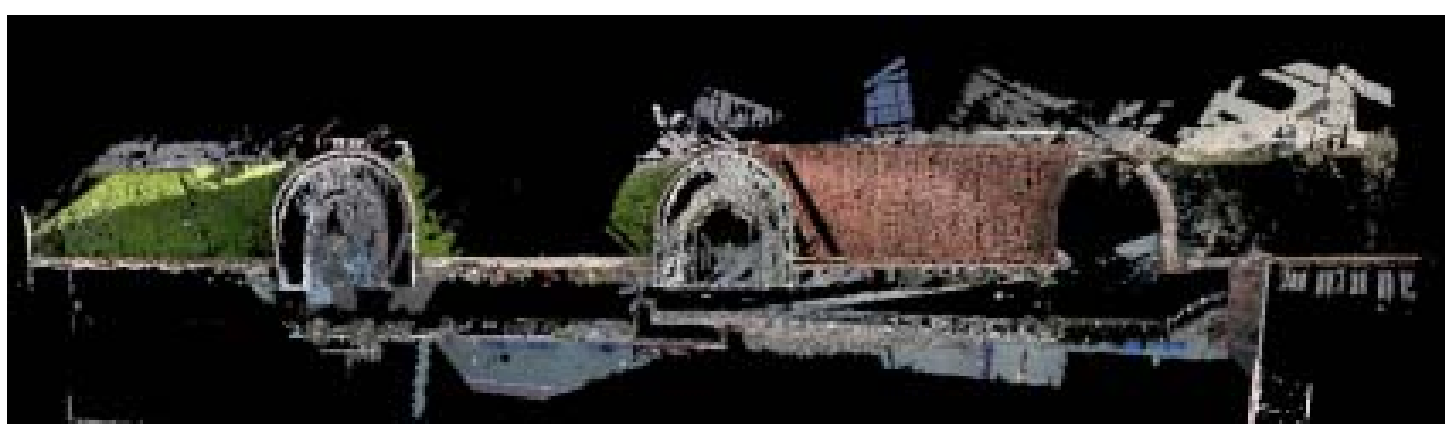

Figura 3: Murallas Romanas y Cercas Medievales de León 
Desarrollar un prototipo que pueda satisfacer los intereses de clientes tan diversos es una tarea ingente que requiere el concurso y la participación de un gran número de expertos. La diversidad de intereses, escala y funciones para los diferentes agentes requiere un enfoque multidisciplinar, multiescala y multifuncional.

\section{TRABAJO EN CURSO}

Actualmente se trabaja en diferentes aspectos del problema de fusión de información $2 \mathrm{~d} / 3 \mathrm{~d}$ y el análisis/visualización de la información que afectan al a) Procesamiento: Extensión al caso 3d de los procedimientos de filtrado y agrupamiento para el tratamiento de la información contenida en nubes de varios cientos de millones de puntos; b) Renderización de objetos: Identificación automática de primitivas geométricas básicas (lineales y cuadráticas a trozos) para automatizar la segmentación volumétrica y extracción de contornos; c) Sistemas de Información con soporte 2d/3d:Conversión de formatos, Detección de hechos, Implementación de procedimientos de búsqueda, acceso y consulta por contenido, Reducción inteligente de la información 3d utilizando muestreo basado en funciones de importancia; d) Documentación arquitectónica y urbana: integración de un módulo de Reconstrucción 3d a partir de múltiples vistas con el módulo de procesamiento de nubes de puntos $3 \mathrm{~d}$ para cubrir huecos relativos a oclusiones situadas en la parte no visible asociada al cono proyectante del láser, especificación y reconocimiento de atributos.

\section{CONCLUSIONES}

La comprensión y descubrimiento de propiedades y relaciones requiere módulos de análisis y visualización que puedan compartir información a diferentes niveles. La gran diversidad de funciones relativas a los sistemas de Información requiere un diseño e implementación de herramientas escalables que puedan proporcionar servicios a diferentes tipos de agentes relacionados con la gestión de espacios urbanos. La plataforma software UVACAD proporciona una respuesta para el análisis y la visualización de información precisa 3d tomando como referencia las nubes de puntos capturadas con láser $3 \mathrm{~d}$.

El desarrollo de un sistema ágil de generación automática de modelos simplificados $3 \mathrm{~d}$ y la simulación de intervenciones sobre escenarios urbanos complejos es nuestro próximo reto a resolver.

\section{Agradecimientos}

Este trabajo ha sido cofinanciado con los fondos de investigación de los Proyectos Nacionales CICYT-MAPA (Modelos y Algoritmos para el Patrimonio Arquitectónico) y Profit-SIPAU (Sistemas de Información para el Patrimonio Arquitectónico y Urbanístico) con soporte del Ministerio de Educación y Ciencia, así como por el Proyecto Regional ARI"Tierras del Renacimiento en Palencia", con soporte de la Consejería de Fomento, Junta de Castilla y León, España. 


\section{Referencias}

J.Finat, M.A..Iglesia Santamaría, J.Martínez-Rubio, J.J..Fernández Martín,J.I..San JoséAlonso and A.Tapias: The Roman Theatre of Clvnia: Hybrid Strategies for applying Virtual Reality on Laser Scanning 3d files", en S.El-Hakim et al (eds): "Virtual Reconstruction and Visualization of Complex Architectures" The Intl. Archives of the ISPRS, Vol. XXXVI, Part: 5/W.17, 2005

J. Finat, J.J.Fernández, J.I.SanJosé, L.Fuentes, J.Martinez and J.D.Pérez-Moneo: "Laserbased 3d GIS for small historical urban zones: Challenges and Results", Intl Conf. and Brookerage Event Construction: Research Needs for the Heritage Protection, Cavtat (Croatia, 2006)

Früh, C. and Zakhor, A, 2004. "An automated method for large-scale, ground-based city model acquisition". Intl J. of Computer Vision, 60(1), 5-20.

Ikeuchi, K., M.Sakauchi, H.Kawasaki, I.Sato, 2004: "Constructing Virtual Cities by using Panoramic Images", Intl J. of Computer Vision, Vol.658 nº 3, 237-247.

Ribarsky, W., 2005: "Virtual Geographic Systems", in C.D.Hansen and J.R.Johnson (eds) "The Visualization Handbook", Elsevier, 449-477.

Teller, S.\& Antone, M., Bodnar, Z., Bosse, M., Coorg, S., Jetwa, M. \& N.Master, 2003: "Calibrated, Registered Images of an Extended Urban Area", Intl. J. of Computer Vision, Vol.53, $\mathrm{n}^{\circ} 1,93-107$.

S.Zlatanova and M.Gruber: "3d Urban GIS on the Web: Data Structuring and Visualizing", D.Fritsch, M.Englisch and M.Sester, eds: IAPRS, Vol.32/4, ISPRS Comm. IV Symposium on GIS, between Visions and Applications, Stuttgart, 2004. 
